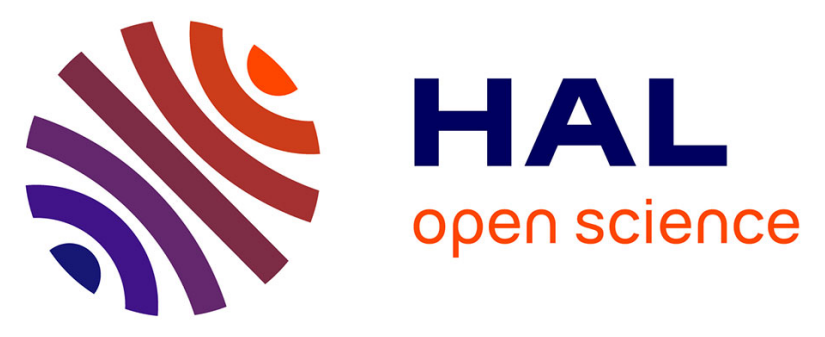

\title{
Building a microfluidic cell culture platform with stiffness control using Loctite 3525 glue
}

Genaro Vázquez-Victorio, Cindy Peto-Gutiérrez, Beatriz Díaz-Bello, Mariel

Cano-Jorge, Daniel Pérez-Calixto, Alejandra Jiménez-Escobar, Silvia

Espinosa-Matías, Reyna Lara Martínez, Rémi Courson, Laurent Malaquin, et al.

\section{To cite this version:}

Genaro Vázquez-Victorio, Cindy Peto-Gutiérrez, Beatriz Díaz-Bello, Mariel Cano-Jorge, Daniel PérezCalixto, et al.. Building a microfluidic cell culture platform with stiffness control using Loctite 3525 glue. Lab on a Chip, 2019, 19 (20), pp.3512 - 3525. 10.1039/c9lc00649d . hal-03041909

\section{HAL Id: hal-03041909 \\ https://hal.laas.fr/hal-03041909}

Submitted on 15 Jan 2021

HAL is a multi-disciplinary open access archive for the deposit and dissemination of scientific research documents, whether they are published or not. The documents may come from teaching and research institutions in France or abroad, or from public or private research centers.
L'archive ouverte pluridisciplinaire HAL, est destinée au dépôt et à la diffusion de documents scientifiques de niveau recherche, publiés ou non, émanant des établissements d'enseignement et de recherche français ou étrangers, des laboratoires publics ou privés. 

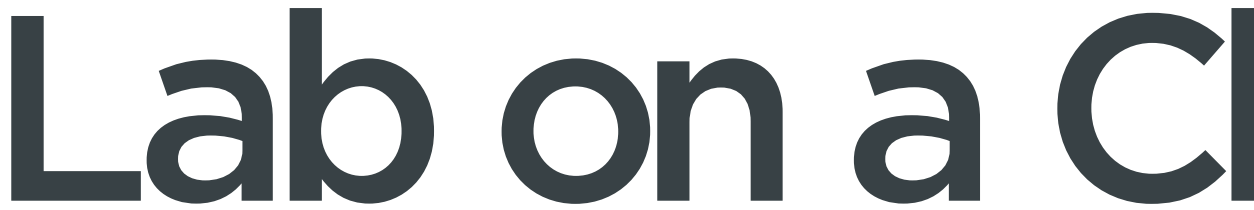

Chip

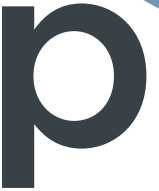

Devices and applications at the micro- and nanoscale

\section{Accepted Manuscript}

This article can be cited before page numbers have been issued, to do this please use: G. Vázquez-

Victorio, C. Peto-Gutiérrez, B. Díaz-Bello, M. Cano-Jorge, D. Pérez.Calixto, A. Jiménez-Escobar, S.

Espinosa-Matías, R. Lara-Martínez, R. Courson, L. Malaquin, D. Zamarrón-Hernández and M. Hautefeuille,

Lab Chip, 2019, DOI: 10.1039/C9LC00649D.
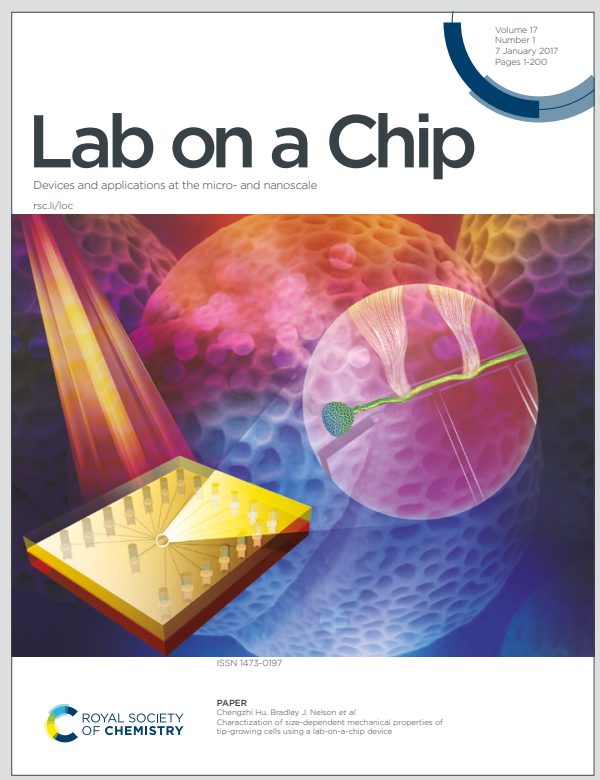

This is an Accepted Manuscript, which has been through the Royal Society of Chemistry peer review process and has been accepted for publication.

Accepted Manuscripts are published online shortly after acceptance, before technical editing, formatting and proof reading. Using this free service, authors can make their results available to the community, in citable form, before we publish the edited article. We will replace this Accepted Manuscript with the edited and formatted Advance Article as soon as it is available.

You can find more information about Accepted Manuscripts in the Information for Authors.

Please note that technical editing may introduce minor changes to the text and/or graphics, which may alter content. The journal's standard Terms \& Conditions and the Ethical guidelines still apply. In no event shall the Royal Society of Chemistry be held responsible for any errors or omissions in this Accepted Manuscript or any consequences arising from the use of any information it contains. 


\title{
Building microfluidic cell culture platform with stiffness controil using Loctite 3525 glue
}

Genaro Vázquez-Victorio, ${ }^{1 *}$ Cindy Peto-Gutiérrez, ${ }^{1}$ Beatriz Díaz-Bello, ${ }^{1}$ Mariel Cano-Jorge, ${ }^{1}$ Daniel Pérez-Calixto, ${ }^{1}$ Alejandra Jiménez-Escobar, ${ }^{1}$ Silvia Espinosa-Matías, ${ }^{2}$ Reyna Lara Martínez, ${ }^{2}$ Remi Courson, ${ }^{3}$ Laurent Malaquin, ${ }^{3}$ Diego Zamarrón-Hernández, ${ }^{1}$ Mathieu Hautefeuille, ${ }^{1^{*}}$

1. Facultad de Ciencias, Universidad Nacional Autónoma de México, Circuito Exterior S/N, Ciudad Universitaria CP 04510, Ciudad de México, México.

2. LAAS CNRS, Université de Toulouse, 7, avenue du Colonel Roche BP 5420031031 Toulouse, France.

${ }^{*}$ Corresponding authors:

genvazquez@ciencias.unam.mx,mathieu_h@ciencias.unam.mx

\begin{abstract}
The study of mechanotransduction signals and cell response to mechanical properties requires designing culture substrates that possess some, or ideally all, of the following characteristics: (1) biological compatibility and adhesive properties, (2) stiffness control or tunability in a dynamic mode, (3) patternability at the microscale and (4) integrability in microfluidics chips. The most common materials used to address cells mechanotransduction are hydrogels, due to their softness. However, they may result impractical when complex scaffolds are sought and they lack viscous dissipative properties that are very important in mechanobiology. In this work, we showed that an off-the-shelf, biocompatible photosensitive glue, Loctite 3525, may be used readily in mechanobiology assays without any special treatment prior to fabrication of cell culture platforms. Despite a high (MPa) stiffness easily tunable by UV exposure time at a fixed dose, 3T3 fibroblasts showed a response to the mechanics of the material similar to that obtained on much softer ( $\mathrm{kPa}$ ) hydrogels. Loctite's viscous dissipation properties indeed seemed to be responsible for such cells mechanical response, as suggested by recent works where more complex two-phase hydrogels were employed. More interestingly, it was possible to stiffen soft Loctite substrates by post-exposing them during cell culture, to observe changes in cell spreading caused by a dynamic stiffness modification. Thanks to Loctite 3525 patternability, micropillars were also fabricated to demonstrate the compatibility with traction force microscopy studies. Finally, the glue was used as an excellent adhesion layer for hydrogels on glass or PDMS, without the need of additional treatment, enabling the readily fabrication of microfluidic chip integrating hydrogels.
\end{abstract}

Keywords: mechanobiology, viscoelasticity, Loctite 3525, rapid-prototyping, microstructuring.

\section{Introduction}


Mechanobiology is an interdisciplinary field that studies the impact of externalvaringacle Online internal physical forces on cellular fate, function, migration and differentiation, caused by the capacity of any cell to sense these forces at the micrometer scale and respond rapidly to these stimuli. Thanks to progress in materials science and microfabrication techniques, it has been possible to demonstrate and investigate the mechanisms through which cells sense the mechanics of their microenvironment and respond to it $1-3$, and also to observe and quantify how much force cells exert on their environment 3 . It is also thanks to technology progress that biomimetic in vitro models have been developed to respond better to a broad variety of specific problems in basic or applied biomedical research where mechanotransduction plays an important role 4 . Understanding biological mechanotransduction processes is now feasible and these important cues are critical to consider when biological processes and mechanisms are investigated.

One of the most important requirements of in vitro mechanobiology studies is the proper selection of the substrates that will be in direct contact with the cells, in particular it is crucial to establish the mechanical properties, responsible for cells adhesion and behavior. The materials need to offer biocompatibility, stiffness control in a physiological range, a possibility to be patterned at the microscale and integrated in a microfluidic chip. It is also critical to design biomaterials and platforms that guarantee high reproducibility, as it is of particular importance when the assays are designed for potential innovative or translational biomedical or healthcare applications 4-6. Among the long list of biomaterials used in mechanobiology, acrylamide-based hydrogels are very common, as they are often good candidates for a biomimetic approach to cell substrate fabrication. Their chemistry enables the attachment of extracellular matrix ligands relatively easily and their typical stiffnesses, in both the elastic and viscoelastic regimes as well as their surface or internal microtopology are capable of reproducing that of in vivo microenvironments characteristics ${ }^{7}$. Although a lot of progress has been made towards standardized fabrication protocols that are now classically used by many laboratories ${ }^{8}$, the level of complexity and number of critical steps in the preparation of the materials, in order to achieve the desired mechanical properties, is a limiting factor. Also, the use of hydrogels may be constrained to static assays on classic culture containers such as glass coverslips or culture plates with no dynamic flows, modification of stiffness or movement. Moreover, the preparation of hydrogels always requires surface pretreatment in order to provide stability in gels attachment for cell culture in medium. There are few examples of the use of hydrogels inside microchips or microfluidic platforms, but they are usually complicated to fabricate as they require many steps and are thus poorly adapted to rapid prototyping 9 .

Thanks to progress in materials science and engineering, it has been possible to understand that adherent cells respond to mechanical environment mainly by the formation of focal adhesions and the subsequent polymerization of actin cytoskeleton 10. The link between the interaction of integrins with extracellular matrix components and the polymerization of actin cytoskeleton has been defined as a clutch model ${ }^{11}$ and it is described as the coupling and transduction of the mechanical properties of 


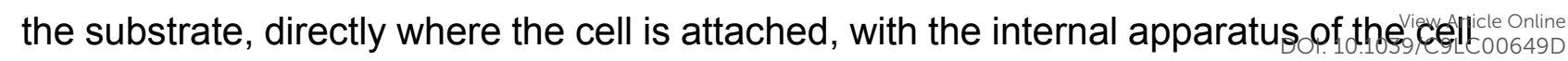
12,13. It has also been shown that actin cytoskeleton polymerizes in stress fibers, to achieve nuclear deformation that is transmitted to nuclear lamina and then promotes rearrangements of several loci to change gene regulation abruptly 14,15 . This mechanical transduction has been demonstrated in mesenchymal stem cells (MSCs) and embryonic fibroblast, as these cells respond strongly to differences in stiffness ${ }^{16}$. Nuclear deformation is also adapted to translocation of transcriptional cofactors. Myocardin related transcription factors (MRTFs) are translocated to nucleus because stress fibers formation (actin polymerization) not only deforms nuclear lamina but it also depletes actin monomers (G-actin) that retain MRTFs at the cytoplasm ${ }^{17}$. This said, YAP, Yes-associated protein, is reported to be the main transcriptional cofactor that transduces mechanical inputs outside the cells ${ }^{18}$. It has been shown that YAP is translocated to nucleus, where it is functional, by actin polymerization. It is not well clear what the mechanism used by F-actin to activate YAP is, but inactivation of LATS kinases is necessary ${ }^{19}$. Recently, it was demonstrated that YAP nuclear translocation is coupled to nuclear lamina deformation because stressing nucleus allows YAP translocate inside nucleus through nuclear pores in mouse embryonic fibroblasts ${ }^{20}$. It is important to analyse focal adhesion formation, actin polymerization and activation of transcriptional cofactors in mechanotransduction studies.

In order to validate biomaterials or platforms for mechanobiology applications, fibroblasts are commonly used, as their response to the stiffness substrate is relatively well-known and this helps identifying or understanding specific mechanistic pathways 21. For instance, it has been shown that stiffness involves a different organization of the cytoskeleton and controls the extent of crosslinked actin filaments inside these cells ${ }^{22}$. Recently, some interesting works have focused on the time response of fibroblasts adhesions to dissipative substrate, showing the great importance of designing materials complying with the characteristic timescales that are responsible for cellular mechanoresponse ${ }^{23,24}$. Spreading in fibroblasts indeed seems to be determined not only by the elastic properties of substrates but also to their viscoelastic properties ${ }^{25,26}$.

In this work, we propose the use of an off-the-shelf material, Loctite 3525 , a commercial UV-sensitive glue, for the direct preparation of cell culture substrates for mechanobiology studies. This commercial material, only previously reported for the fabrication of microfluidics patterns and chips ${ }^{27}$, has been readily used to replicate micromolds features with great fidelity and may even be directly patterned at microscale using a blu-ray laser ${ }^{28}$. We show here that this photosensitive resin offers an interesting alternative as a biocompatible, transparent material for mechanobiology studies with high reproducibility in fabrication, simplicity of use, great accessibility and low-cost. We first demonstrated that a stiffness tuning is possible by controlling the crosslinking via UV (365 nm) exposure time and we found great differences in the $2 \mathrm{~s}$ to 40 s range. In spite of a high elastic modulus of the cured material (5 - $25 \mathrm{MPa}$ range), wee observed that 3T3-L1 mouse embryonic fibroblasts seeded on Loctite 3525 layers responded differently to different exposure times, evidenced by very different spreading areas. The measured cell areas of the fibroblasts were found to be 


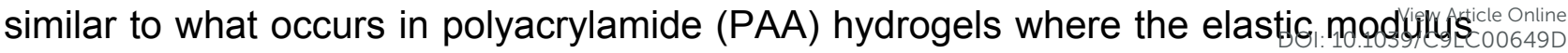
was measured in the typical reported range of $\mathrm{kPa}$. To explain this abnormal behavior, confirmed by YAP/TAZ localization in spread cells, and after verifying that it could not be attributed to differences in surface chemistry, we then characterized the relaxation modulus of the cured material to define the viscous dissipations that were reported to be a possible cause for apparent, immediate stiffness, as viscoelasticity could modify greatly the behavior of 3T3-L1 cells, even more than elastic modulus alone, according to recent reports in ${ }^{24}$ and ${ }^{25}$. After demonstrating that Loctite 3525 is very useful to study mechanotransduction as its viscoelastic properties emulate soft elastic hydrogels, the less-crosslinked Loctite samples were further cured after fibroblasts were cultured on them, in order to induce a dynamical modification of the mechanical properties during culture and test dynamic mechanotransduction processes inside the cell while stiffness is increased, similar to ${ }^{25,29}$. Loctite 3525 has also been used in this work as a 3D microstructured material to control microtopology in cell culture. In particular, we tested micropillars fabrication for studies in traction force microscopy to measure cell force using such patterns ${ }^{30}$. Finally, cured Loctite 3525 was employed as a very strong adhesion layer for hydrogels, stable in cell culture media, without the need for any additional surface treatment. We found that the material may even be cured on plastics, PDMS and glass substrates onto which it adhered very well and then hosted hydrogels without further pre-treatment. This is particularly important when mechanobiology assays are required in PDMS microfluidic chips where low Young's moduli are required ${ }^{9}$.

\section{Experimental section}

Materials: light-cure acrylic-based Loctite 3525 adhesive was purchased from Henkel company. Polydimethylsiloxane (PDMS) consisted of a Sylgard 184 kit, obtained from Dow Corning $\mathbb{R}$. Poly- $\square$-lysine wt $>30,000$, acrylamide, N,N'Methylenebis(acrylamide), ammonium persulfate TEMED, acrylic acid $\mathrm{N}$ hydroxysuccinimide (NHS) ester and Irgacure 2959 were purchased from SigmaAldrich. Culture reagents and media were obtained from ThermoFisher Scientific. Rat tail type I was obtained from Corning $\mathbb{R}$. Calcein AM, Alexa Fluor 488 phalloidin and anti-mouse secondary antibody and DAPI were obtained from Molecular Probes. AntiYAP monoclonal antibody (sc-101199) was obtained from Santa Cruz Biotechnology. Focal Adhesion Staining Kit (FAK100) and Mowiol was purchased from Millipore.

Cell lines and culture conditions: mouse embryonic fibroblasts (3T3-L1, a gift from Dr Marina Macías-Silva at Instituto de Fisiología Celular, UNAM) were cultured in Dulbecco's Modified Eagle's Medium (DMEM) complemented with $10 \%$ of fetal bovine serum (FBS) plus penicillin/streptomycin antibiotics. 1.5 to $3.0 \times 10^{4}$ Fibroblast were cultured over a Loctite-covered $20 \mathrm{~mm}$ round coverslip in 12-well plates. Human lung adenocarcinoma cell line (A549, a gift from Dr José G. Cisneros-Lira at Instituto Nacional de Enfermedades Respiratorias) was cultured in F-12 Medium 
complemented with $10 \%$ of FBS plus antibiotics. Human liver hepatocellivitaricle Online carcinoma cell line (HepG2, HB-8065 ATCC) was cultured with Minimal Essential Medium (MEM) complemented with $10 \%$ of FBS and $1 \%$ of sodium pyruvate plus antibiotics. A549 cells were seeded at a confluence of $1.5 \times 10^{4}$ cells per PAA-covered round coverslips. For culturing the chip channel, HepG2 cells were seeded at a confluence of $1.5 \times 10^{7}$ cells $/ \mathrm{ml}$. All cell lines were cultured at standard conditions of $5 \% \mathrm{CO} 2$ and $37^{\circ} \mathrm{C}$.

Immunofluorescence assays: for Live/dead assay, 3T3-L1 fibroblasts and HepG2 cell line were incubated in starvation media (without FBS). [1 $\mu \mathrm{M}]$ Calcein AM and $[5 \mu \mathrm{M}]$ propidium iodide $(\mathrm{PI})$ were incubated for $15 \mathrm{~min}$ before previous detection. For apoptotic cells detection, 3T3-L1 fibroblasts were fixed after $48 \mathrm{~h}$ of culture in $1 \%$ of paraformaldehyde (PFA) in PBS $1 \mathrm{X}$ for $10 \mathrm{~min}$ at room temperature (RT) and then cells were post-fixed in pre-chilled $2: 1$ ethanol:acetic acid solution at $-20^{\circ} \mathrm{C}$ for $5 \mathrm{~min}$. Following this, the samples were processed according to the manufacturer's instructions for Apoptag ${ }^{\circledR}$ fluorescein in situ apoptosis detection kit (Merck). 3T3-L1 fibroblasts and A549 cell line were fixed with $4 \%$ of PFA in PBS for 15 min at $37^{\circ} \mathrm{C}$. Cells were permeabilized with $0.1 \%$ of Triton $X-100$ for 5 min and blocked with $0.1 \%$ of albumin plus $10 \%$ of horse serum in PBS (blocking solution) for $1 \mathrm{~h}$ at RT. For actin cytoskeleton detection, we incubated Alexa Fluor 488 phalloidin in PBS diluted 1:50 for $30 \mathrm{~min}$ at RT. For focal adhesion detection, we used monoclonal antibody against vinculin and Alexa Fluor 594 phalloidin both diluted 1:100. We detected YAP/TAZ proteins by using anti-YAP diluted 1:100. All primary antibodies were incubated in blocking solution overnight at $4^{\circ} \mathrm{C}$ and secondary antibodies were incubated in a dilution of 1:500 in blocking solution for 1-2h at RT. Nuclei were stained by using propidium iodide $(\mathrm{PI})$ due to Loctite 3525 resin fluoresces strongly at UV wavelength spectrum. All samples were mounted in Mowiol mounting resin for preservation (Merck). Epifluorescence microscopy (Nikon Eclipse) and confocal microscopy (Leica TCS SP8) were used for image acquisition. Image analysis and edition was done by using Free Fiji software. A Student's t test was done to calculate statistical significance using Prisma software and $p$ value $<0.05$ was considered to be significant.

PAA polymerization on Loctite 3525 substrates: polyacrylamide (PAA) hydrogels were reticularized according to a protocol described previously in ${ }^{31}$. In brief, we mixed $40 \%$ acrylamide and $2 \%$ bis-acrylamide solutions in specific proportions to achieve desired elastic values. We added ammonium persulfate and TEMED for acrylamide polymerization and incubated the mixture for $15 \mathrm{~min}$ on the Loctite-covered round coverslips. [100 $\mu \mathrm{g} / \mathrm{mL}$ ] Rat tail collagen type I was crosslinked to PAA by mixing collagen with acrylic acid NHS ester and Irgacure 2959 and exposing the mixture to $365 \mathrm{~nm}$ wavelength UV (UVP crosslinker CL-1000L).

\section{Results and discussion}


Loctite $\mathbf{3 5 2 5}$ as a substrate material for mechanobiology Polymerization of Loctite 3525

Loctite 3525 is a commercially available UV-sensitive material that offers great depths of cure, down to a centimeter, under little exposition irradiance $\left(30-50 \mathrm{~mW} / \mathrm{cm}^{2}\right)$ at 365 $\mathrm{nm}$ according to its datasheet and our own characterization (see Supporting Information Material Figure S1). It is viscous (9 to 31 Pa.s according to the datasheet), transparent and very easy to use thanks to its syringe applicator. Although little information is available about its composition, the Material Safety Datasheet states that it is a methacrylate-based resin: it contains mainly polyurethane methacrylate resin, 2-hydroxyethyl methacrylate and high boiling methacrylate. The MSDS information also states that it uses diphenyl-2,4,6-trimethylbenzoyl phosphine oxide (commonly known as TPO) as photoinitiator. This type of very poorly soluble photoinitiator is typically used in the range of $1-5 \%$ (also found in the MSDS composition table in this proportion) in unsaturated polyesters and resins containing acrylic ester groups and permits a rapid polymerization process as it divides into two free radicals, which are then incorporated into the polymer as chain ends. No residual TPO is normally found in the materials where it is used as it is captured in the polymer matrix that is crosslinked rapidly.

In order to guarantee reproducibility, a nanoimprint lithography (NIL) system, Eitre 3 model from Obducat, was used to prepare flat layers of Loctite 3525 of controlled height and avoiding superficial roughness. Indeed, this system uses $365 \pm 10 \mathrm{~nm}$ LEDs to homogeneously irradiate UV at a fixed dose of $80-100 \mathrm{~mW} / \mathrm{cm}^{2}$ onto a sample placed in its sealed chamber. The control of the soft pressure applied upon the sample protected by a transparent acetate sheet enabled by the system allowed for a complete flatness of the Loctite surface during crosslinking and the control of the layer thickness. This particular feature is critical to discard the influence of any surface pattern in cell spreading response and thus attribute the following observations to mechanics.

Polymerization of the Loctite 3525 samples cured at different exposure times was verified using Attenuated Total Reflectance Fourier Transform Infrared Spectroscopy (ATR-FTIR), using a Perkin Elmer Spectrum Two system on samples treated for cell culture. Almost no differences were found between the spectra of the different exposure times in the materials except for a small increase of the transmission of the $816 \mathrm{~cm}^{-1}$ centered band relative to the $845 \mathrm{~cm}^{-1}$ peak (Supporting Information Material Figure S3). This band relation is typically monitored to assess polymerization of acrylate resins ${ }^{32}$ and it showed here that crosslinking was not complete after only $2 \mathrm{~s}$ of exposure but all spectra after $4 \mathrm{~s}$ were similar, demonstrating that no further material modification could be found after longer exposure times and curing was complete. All spectra showed an excellent consistency between runs, proving that control of UV dose and exposure time could enable regulation of curing, as expected. Also, as depicted in Figure S2, no other surface chemistry differences were observed between differentially polymerized Loctite samples, for instance no amine bands were identified. It was then relevant to test the correlation between the degree of Loctite curing and its mechanical properties. 
Mechanical properties of the UV-exposed material were then tested using a microindenter (Femto Tools, MTA-02 system) with a spherical glass tip of a radius $\mathrm{R}_{\text {tip }}$ of $25 \mu \mathrm{m}$ (Femto Tools, FT-S10000) on 5 locations of at least 5 samples of each of the curing types, $2 \mathrm{~s}$ and 40 s exposure dose. We have tested not only dry samples (in air) but also wet measurements (in culture medium), in order to better mimic the ambient conditions experienced by cells in their culture medium. After indenting inside the Loctite layers and measuring the resulting force, the Hertz model was used in order to determine the elastic Young's modulus of all samples; in this case, we limited the analysis and calculation to indentation depths of down to $0.3 \times \mathrm{R}_{\text {tip }}$ and used a Poisson ratio value of $0.2233,34$. For all exposition times tested in our experiments, the calculated value of Young's modulus consistently fell between 25 and 40 MPa for all tested samples in the dry condition, suggesting that the material was always cured (the manufacturer datasheet reports a ultimate tensile strength value of $25 \mathrm{MPa}$ ). As expected with a thin Loctite 3525 layer and the $80 \mathrm{~mW} / \mathrm{cm}^{2}$ dose employed here, longer irradiance times do not seem to stiffen the material further. We decided to use the extreme $2 \mathrm{~s}$ and $40 \mathrm{~s}$ in all the following experiments and Figure 1.A shows the obtained results. As suggested by the previous FTIR measurements, the samples cured with $2 s$ presented a lower average stiffness than all other materials. The influence of medium on the material mechanical properties was confirmed experimentally, as shown in Figure 1.A: the stiffness of the samples decreased in aqueous medium. Interestingly, the 2s-exposed samples presented a Young's modulus very similar to that of 40s exposure in dry conditions but it resulted 4 times lower in medium between the two wet samples (5.48 MPa in average versus $20 \mathrm{MPa}$, respectively).
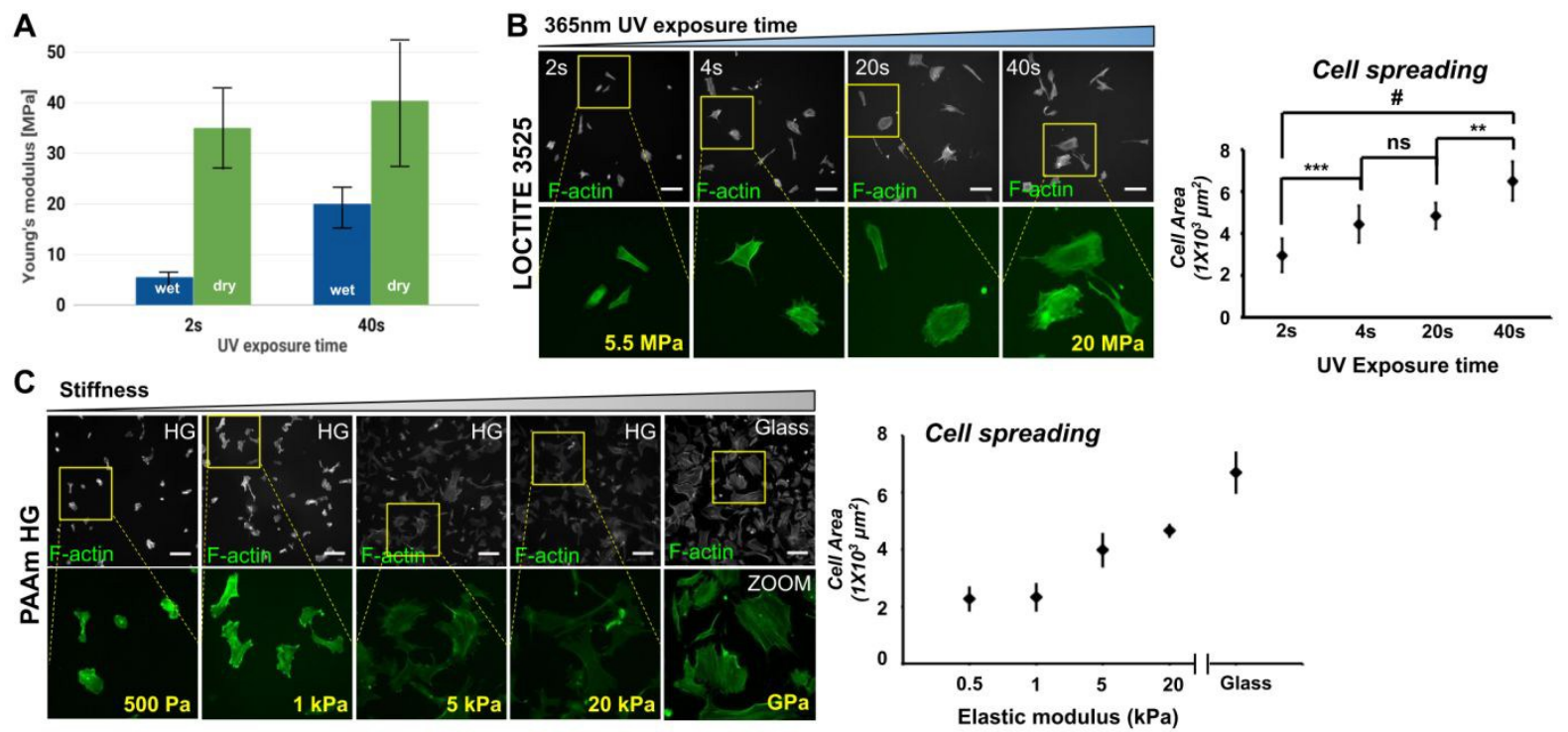

Figure 1. Loctite 3525 as a mechanobiology material. (A) Control of Young's modulus with UV exposure time at $365 \mathrm{~nm}$, obtained from indentation measurements fit using the Hertz model. (B) Spreading of 3T3-L1 mouse embryonic fibroblasts cultured on differentially cross-linked Loctite 3525 
for 24 hours. Loctite was exposed with $365 \mathrm{~nm}$ UV light for 2, 4, 20 and 40 seconds in aNIL Ejitresesicle Online system. F-actin was detected by Alexa Fluor 488 phalloidin (green). Cell spreading analysis was presented as the area detected by phalloidin in 3T3-L1 fibroblast cultured on differentially cross-linked Loctite 3525. Data are represented as mean \pm SD of 5 different fields of a representative of 3 independent experiments. ${ }^{* * *} p<0.0003$ and $\# p<0.0008$ compared with $2 \mathrm{~s} ;{ }^{* *} p<0.0076$ compared with $20 \mathrm{~s} ; n s$, not significant. (C) Polyacrylamide hydrogels (PAA HG) were polymerized to obtained different stiffness values from $0.5,1,5$ and $20 \mathrm{kPa}$. Glass coverslips were used as a control of maximum cell spreading level (GPa). F-actin was detected by Alexa Fluor 488 phalloidin (green) and cell spreading analysis was presented as the area detected by phalloidin in 3T3-L1 fibroblast cultured for 24 hours on differentially cross-linked PAA HG. Data are represented as mean \pm SD of at least 3 different fields a representative of 3 independent experiments. Scale bar is $200 \mu \mathrm{m}$.

\section{Fibroblast mechanobiology on Loctite 3525}

After setting the polymerization conditions to control Loctite stiffness, we used mouse embryonic fibroblasts 3T3-L1 cell line, as this cell line has been shown to respond well to the mechanical stimulus of the substrates ${ }^{35,36}$. We seeded $3 T 3$ fibroblast onto pristine, cured resin immediately after polymerization, but cells did not adhere to Loctite without any adhesion promoter (data not shown). All fabricated substrates were thus coated with poly-L-lysine with a molecular weight $>30000 \mathrm{Da}$ in order to promote a strong cell adhesion and ensuring that the spreading of the cell was totally dependent on the mechanical properties of Loctite and not caused by weaknesses in surface binding ${ }^{37}$. It is important to highlight that the addition of adhesion promoters did not require additional UV exposure or previous chemical modification as some other surfaces do ${ }^{38}$. Interestingly, we observed that fibroblasts adhered efficiently to all the samples for each curing time that was tested (data not shown). However, after 24 hours of culture, $3 \mathrm{~T} 3$ fibroblasts showed differences in spreading on the different sample types, that correlated well with UV exposure times (Figure 1.B). We also assessed F-actin formation in fibroblasts cultured on Loctite, because it is known that actin polymerization is needed to reinforce the spreading process ${ }^{22,30}$. It was found that both the increase of cell total area and appearance of F-actin were dependent of UV light exposure (Figure 1.B). Quantification of changes in cell area shown a 2-fold increase between $2 \mathrm{~s}$ and 40 s of polymerization (2,958 versus 6,492 square microns, respectively) suggesting strongly that the differences in Loctite cross-linking is causing differences in mechanical properties of the material that is sensed by the fibroblast. The effect observed here has been demonstrated not only for fibroblasts but it seems to be ubiquitous ${ }^{39,40}$. Remarkably, this behavior was not expected for 3T3 cells in the $\mathrm{MPa}$ range, measured here by indentation, as a spreading normally occurs as a mechanotransduction response in the $\mathrm{kPa}$ range, reaching an asymptotic plateau around a few hundreds of $\mathrm{kPa}$ and up to the GPa range ${ }^{22}$. Moreover, the difference in the measured elastic moduli between $2 \mathrm{~s}$ and 40 s exposure seemed not significant enough to justify such a difference in shape and area between the two materials. Experiments of cell spreading on soft elastic PAA hydrogels (with controlled stiffness in the $\mathrm{kPa}$ range) and stiff glass coverslips (GPa) were performed as soft and stiff 


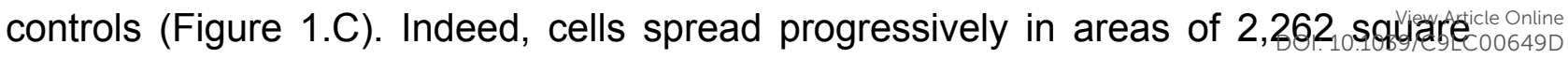
micrometers per cell for 500Pa and 6,695 square micrometers per cell for glass. Very interestingly, the fibroblasts spreading found on Loctite samples that were progressively crosslinked was similar than what was seen on the hydrogels $(2,326$ and 4,660 square micrometers for $1 \mathrm{kPa}$ and $20 \mathrm{kPa}$, respectively), although the elastic modulus of the gels was three orders of magnitude lower than that measured for Loctite, as depicted in Figure 1.C. We show the results obtained for crosslinking durations of $2 \mathrm{~s}, 4 \mathrm{~s}, 20 \mathrm{~s}$ and $40 \mathrm{~s}$ UV exposure in order to demonstrate the progressive cell spreading on Loctite in the MPa range (Figure 1.B) comparable to what is typically found for the same fibroblasts on kPa-range stiffnesses on hydrogels. However, as mentioned earlier, only the extreme $2 \mathrm{~s}$ and $40 \mathrm{~s}$ were used in the following mechanotransduction studies.

It is important to remind that no difference in material surface chemistry was found between the different Loctite samples in the FTIR spectroscopy characterization and we studied if such a spreading behavior is a mechanical response of the 3T3 fibroblast mechanotransduction response. In order to try to confirm this hypothesis, we focused on differential cell responses between $2 \mathrm{~s}$ and 40 s cross-linked Loctite samples, because the difference in spreading was significant. First, we examined cell confluence on these two conditions during 48 hours after culture. We observed that fibroblasts cultured on 40s-exposed Loctite samples presented a higher number of cells compared with the 2s-cured resin. Quantification of cell number showed an increase of 1.7 fold (104 vs 176 cells per area) between the substrates (Figure 2.A). In order to discard the role of cytotoxicity in our observations, we tested cell viability in 3T3-L1 fibroblasts cultured on Loctite exposed for $2 \mathrm{~s}$ and 40s under a $365 \mathrm{~nm}$ UV source in order to exclude apoptosis induction of the cured resin (as shown in Supporting Information Material Figure S8). It is clearly evident that there was no apoptosis induction by DNA strand breaks in any cured resins discarding the possibility of toxicity effects of cured Loctite and possible loosely crosslinked parts of the material that would eventually surface out when placed in aqueous medium. This observation correlates in that there is no evidence about the induction of DNA damage by alkyl methacrylates such as 2-Hydroxyethyl methacrylate, an important component of Loctite 3525 resin ${ }^{41}$. So, it is thus likely that the difference in cell number is due to cellular proliferation rather than cell death, however, further studies must be done to be conclusive. It has been reported that there is a strong relationship between stiffness and proliferation rates in fibroblasts. Cell proliferation is dependent on cell spreading that is regulated by the mechanical properties of substrates and its direct impact on intracellular actomyosin tension ${ }^{42,43}$. The differences in cell spreading and cell number observed between the two substrates suggested that the cell response to mechanics could be caused by differences in the establishment of focal adhesions over the substrates due to the mechanical differences ${ }^{44}$. We detected focal adhesions (FA) by detecting vinculin, that is an essential component of FA. As expected, we observed a higher density of vinculin signal in FA on 40s-exposed Loctite samples, suggesting that the differences observed in our experiments could be explained by the maturity on focal adhesions (Figure 2.C, heat map). We used vinculin as a marker to visualize 


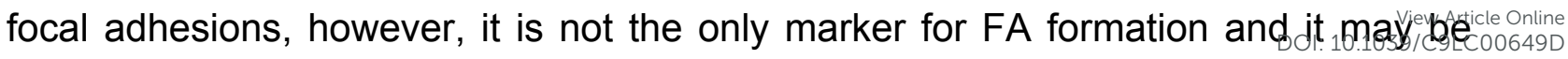
possible that other proteins involved in FA maturation could show differences in recruitment ${ }^{45}$. Additionally to FA formation, we decided to study how cells responded downstream and analyzed the influence of fibroblasts spreading in the activation of mechanosensitive transcriptional cofactors, YAP and TAZ. Indeed, YAP/TAZ proteins are translocated to nucleus and this activation depends on cell spreading and actin polymerization on stiffer substrates ${ }^{18}$. Here, we used glass coverslips as a much stiffer substrate for control (GPa range), in order to achieve the highest YAP/TAZ nuclear localization and compare with 2 s and 40 s exposed samples ${ }^{46}$. Again, as expected, the nuclear translocation of YAP/TAZ proteins was promoted as the stiffness of Loctite was increased by UV dose exposure time in cultured fibroblasts. Quantification of the cytoplasmic and nuclear localization of YAP/TAZ proteins is shown in Figure 2.C. It revealed at least a 2-fold decrease in the levels of YAP/TAZ proteins inside the nucleus compared to cytoplasm ( $31 \%$ and $14 \%$ for 40 s and 2 s UV exposed Loctite, respectively). These results seemed to confirm a mechanosensitive response of 3T3 fibroblasts caused by the spreading on differentially exposed Loctite samples. Additionally, we detected ß-catenin protein signal in Loctite samples exposed for $2 \mathrm{~s}$ and 40s. In glass, ß-catenin protein was clearly localized in cell junctions, however, the signal density decreased in both UV exposed samples (as shown in Supporting Information Material Figure S9). Besides the decrease in signal density in cell junctions, we observed a cytoplasmic localization of ß-catenin in fibroblasts on $2 \mathrm{~s}$ cured Loctite samples. These results are consistent with previous data that showed that $ß$-catenin signaling is involved in adipose and osteogenic differentiation in embryonic cells that is strongly related with substrate stiffness 47,48 .

A
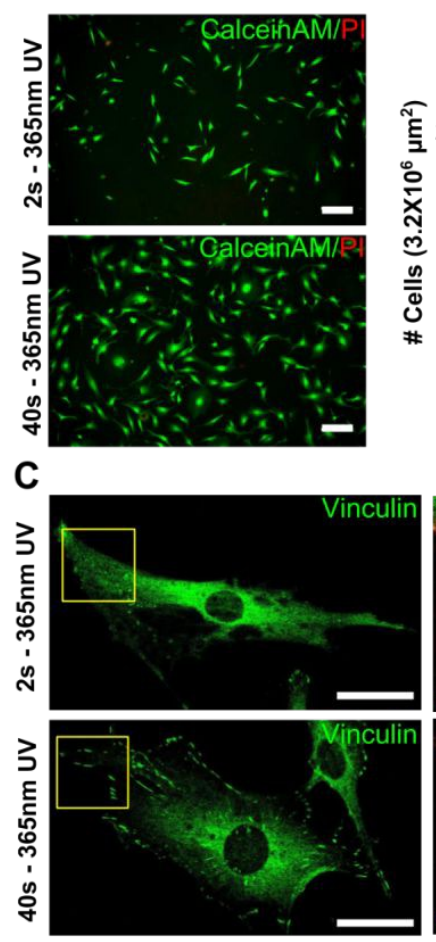

48h of Culture
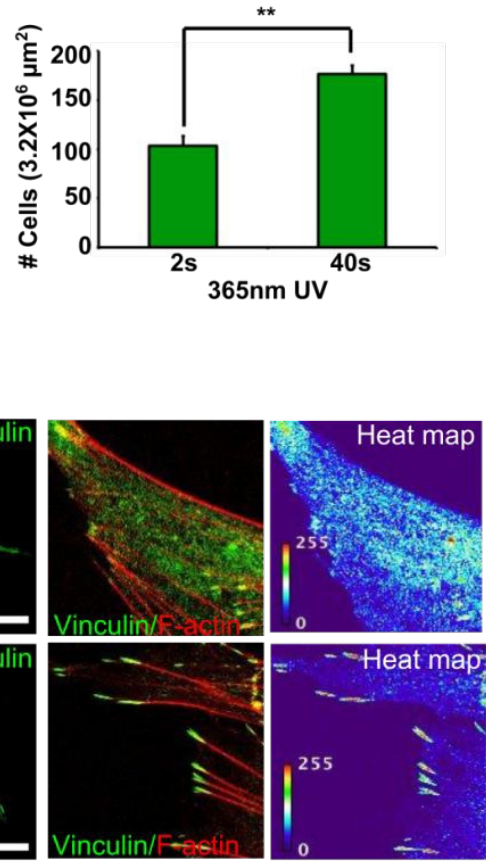

B

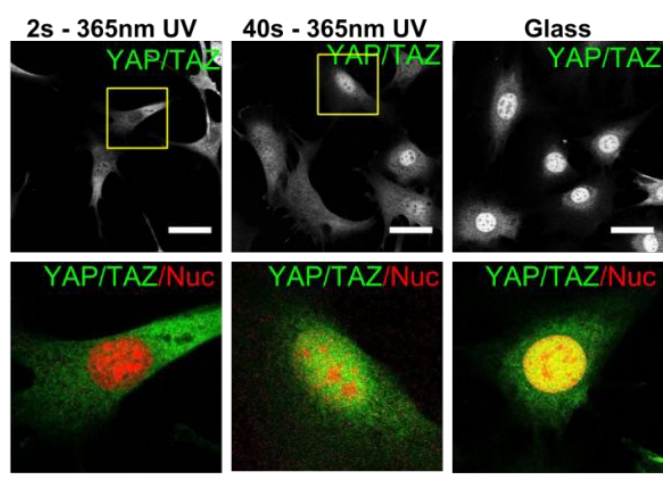

YAP/TAZ subcellular localization $\square$ NUC $\square$ CYTO

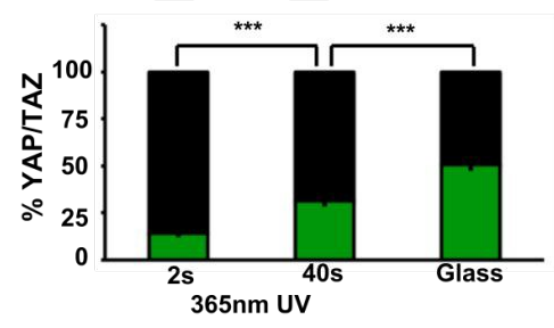


Figure 2. Fibroblasts sense mechanical properties of differential polymerized Loctite $3525.15039 / 52$ Viecle Online Live/dead assay of 3T3-L1 mouse embryonic fibroblasts. Fibroblasts were cultured at 2 and 40s UV exposed Loctite 3525 and cells were incubated with $[1 \mu \mathrm{M}]$ of calcein $\mathrm{AM}$ and $[5 \mu \mathrm{M}]$ of propidium iodide (PI) for 15 min 48 hours after culturing. Cell count was presented as a total number of cells in an area of $3.2 \times 10^{6} \mu \mathrm{m}^{2}$. Data are represented as mean $\pm \mathrm{SD}$ of 12 different fields from 2 independent experiments. Scale bar $=100 \mu \mathrm{m} .{ }^{* *} p<0.0052$ compared with $2 \mathrm{~s}$. (B) Nuclear translocation of YAP/TAZ mechanosensitive cofactors in fibroblasts cultured on differential cross-linked Loctite 3525. Fibroblast cultured on glass coverslips were used as control for full YAP/TAZ nuclear translocation. YAP/TAZ subcellular localization was detected by immunofluorescence (green); nucleus was detected by PI (red). YAP/TAZ subcellular localization was presented as the percentage of nuclear and cytoplasmic localization. Data are represented as mean $\pm \mathrm{SD}$ of at least 4 different fields of a representative of 2 independent experiments. Scale bar $=50 \mu \mathrm{m}$. $* * * p<0.0001$ compared with $2 \mathrm{~s}$; *** $p<0.0002$ compared with 40s. (C) Focal adhesions (FA) of fibroblast cultured on differential UV cured Loctite 3525. FA were detected by immunofluorescence using focal adhesion detection kit. Vinculin (green); F-actin (red). Heat map of vinculin signal calibrated from 0 to 255.

What was found surprising in the experimental results is that the mechanoresponse of the 3T3 fibroblasts cultured on Loctite 3525 identified by an increase in measured cell areas is comparable with what has been reported elsewhere and in this work on much softer elastic materials, in the stiffness range of $\mathrm{kPa}$ and was not expected in the MPa range.

\section{Viscoelastic properties of Loctite 3525}

In order to explain the observed phenomenon, the viscoelastic properties of the glue were studied using the same microindenter system and configuration. Indeed, it has been shown recently that the presence of a viscous material inside the elastic matrix may have a much greater impact than elasticity and Young's modulus on cell spreading 25,26. Also, the relevance of performing Young's modulus calculations from indentations in order to determine the mechanical properties sensed by the cells at microscale appeared to be questionable in this type of experiment: several recent works reported having identified the actual combinations of relevant mechanical properties responsible for cell spreading and calculate their values at the scale of the $1 \mu \mathrm{m}$-sized focal adhesions ${ }^{24,25}$. Even though our polymerized resin presented a much larger elastic modulus (several orders of magnitude) than what was reported for hydrogels, it is possible that the mechanism responsible for the absence of cell spreading on the 2s-exposed Loctite layers is related to viscosity. Moreover, the adhesion sites are always small on dissipative substrates, as suggested in the literature ${ }^{24}$ where, similar results were observed and 3T3 cells maintained a rounded morphology on $5 \mathrm{kPa}$ hydrogels if a small fraction of viscous phase was found in the material. It was argued that by decoupling viscoelastic characteristics from elastic ones, it may thus be possible to modulate viscoelastic relaxation dynamics while keeping the equilibrium elastic modulus constant and thus control cell traction forces, spreading and phenotype. Following the idea that the viscoelasticity of the substrates has to be tuned to control cell spreading, another recent work showed that it is possible 
to model cell spreading speed on viscoelastic substrates by measuring the evoluffitenticle Online of relaxation stiffness over time and fitting this material relaxation response to a generalized Maxwell model. This standard linear solid (SLS) model consists of several springs-dashpots (additional stiffness $k_{\mathrm{a}}$ of the spring and viscosity $\eta$ for the dashpots) coupled in parallel with the long-range stiffness $k_{l}$ of the elastic part of the material, responsible for the slow relaxation of viscoelastic materials ${ }^{25}$. Interestingly, they showed that cell spreading is controlled by two important conditions: the relationship between the relaxation timescale $T_{\mathrm{s}}=\eta / K_{\mathrm{a}}$ of the viscous phase of the material and the binding time of the cell with its substrate $\tau_{\mathrm{b}}$ (or clutch binding timescale, responsible for focal adhesions, in the order of 1 second) and the impact of the additional stiffness $k_{\mathrm{a}}$ of this viscous phase ${ }^{22}$.

Following our observations and basing our analysis on these results to explain the result, further mechanical tests were performed to characterize better our materials. Moreover, it was observed that Loctite 3525 swelled slightly in aqueous solutions, demonstrating a certain tendency to absorb cell culture medium that could influence its mechanical properties. The relaxation stiffness of our samples was then measured by indenting at fixed depth and measuring force as a function of time (Figure 3). The relaxation stiffness was then modelled using the generalized Maxwell model and all relevant mechanical properties, including the significant $t_{\mathrm{s}}$ timescales, of both soft and stiff Loctite materials were calculated using the method reported in ${ }^{25}$. These results are shown in Table 1 (and calculation details are given in the Supporting Information Material). First, it is interesting to note that the difference between the relaxation behaviors of the two materials is demonstrated by the two significant timescales ts and the long-time equilibrium stiffness values that related well to the experimental results shown in Figure 3. First, the significant timescale of the 40s-exposed sample is much closer to the clutch binding timescale of 1s, a sign that a 3T3 fibroblast on such substrate would spread more than on a 2s-exposed sample according to ${ }^{25}$. Then, it is very interesting to note that the viscosity of both samples is similar, but their relaxations behaviors are very different, both in time and in relative amplitude, and this is explained by the difference in their immediate $k_{\mathrm{a}}$. When analyzing this particular result with the model detailed in ${ }^{25}$, it is clear that spreading will occur much more on the longer-exposed Loctite substrates than on the others, regardless of the value of the equilibrium elastic modulus E'. Indeed, as presented in Figure 2 and Figure S5 based on previous works ${ }^{25}$, a lower value of $k_{a}$ prevails in the instantaneous elastic modulus $E=E^{\prime}+E^{\prime \prime}$ sensed by the cells to avoid spreading and the model definitely predicts a much greater and faster spreading for the material obtained after 40s of UV light exposure. Moreover, although both the storage and loss moduli (calculated in our materials considering a $1 \mu^{2}$ focal adhesion) were found in the hundreds of $\mathrm{kPa}$ range, they are still at least 40 times greater than what was observed in ${ }^{24}$ and it remains surprising that cells spreading is caused by a relaxation time of partially or fully cured Loctite. This suggests that further experiments are required to look for the viscous part in 2s-exposed Loctite and for possible predominant interactions of cells with this viscous phase at the surface. It is also possible that the time-dependent viscoelastic properties of the material are caused by an internal interaction of the 


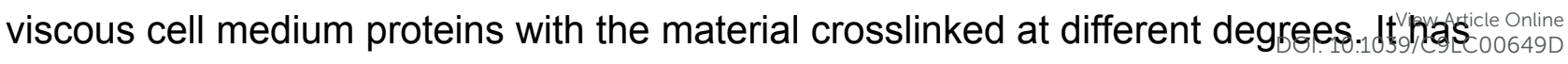
indeed been shown very recently that the viscosity of the liquid phase of crosslinked polyacrylamide and agarose hydrogels could be substantially modified without altering the elastic component, by preparing the gels in aqueous solutions of dextran of different concentrations ${ }^{49}$. However, although the underlying mechanism responsible for this observed mechanoresponsive behavior of 3T3 cells remains unknown in the MPa range, we have shown that Loctite 3525 is an excellent viscoelastic material to study cellular mechanoresponse, without the need to prepare complex composite hydrogels with different phases. It has proven to be highly reproducible and the control of relevant mechanical parameters is only achieved by UV light exposure time.
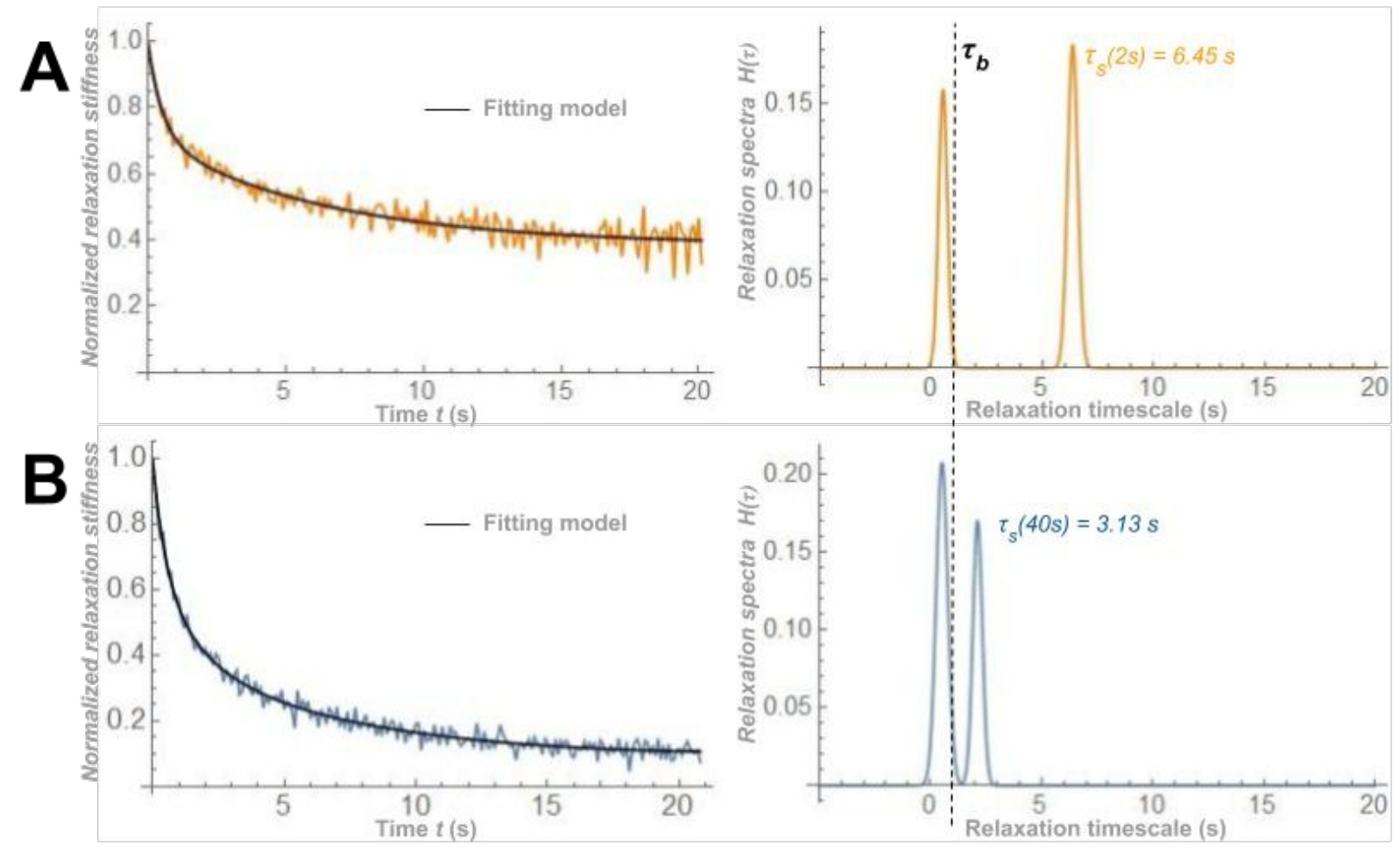

Figure 3. Normalized relaxation stiffness of cured Loctite layers as a function of time with the fitting model (left) and corresponding relaxation spectra showing relevant timescales (right) for 2 s-exposed samples (A) and 40s-exposed ones (B). Only the most significant timescales close to the binding timescale are shown.

Table 1. Mechanical properties of Loctite 3525 averaged from relaxation measurements obtained with a microindenter with samples placed in cell medium $(\mathrm{n}=3)$.

\begin{tabular}{|c|c|c|}
\hline Mechanical properties & 2s exposure & 40s exposure \\
\hline stiffness $\boldsymbol{k}_{\boldsymbol{\theta}}[\mathrm{pN} / \mathrm{nm}]$ & $0.22 \pm 0.01$ & $0.38 \pm 0.04$ \\
\hline relaxation timescale of viscous phase $\boldsymbol{\tau}_{\boldsymbol{s}}[\mathrm{s}]$ & 6.45 & 3.13 \\
\hline
\end{tabular}




\begin{tabular}{|c|c|c|}
\hline long-range stiffness $\boldsymbol{k}_{\boldsymbol{l}}[\mathrm{pN} / \mathrm{nm}]$ & $0.17 \pm 0.01$ & $\begin{array}{r}\text { View Artic } \\
0.28 \pm 0.059 / \mathrm{C} 9 \mathrm{LC}\end{array}$ \\
\hline additional (immediate) stiffness $\boldsymbol{k}_{\boldsymbol{a}}[\mathrm{pN} / \mathrm{nm}]$ & $0.05 \pm 0.001$ & $0.1 \pm 0.02$ \\
\hline viscosity of the dashpot $\boldsymbol{\eta}\left[\mathrm{pN}^{*} \mathrm{~s} / \mathrm{nm}\right]$ & 0.34 & 0.32 \\
\hline tensile storage modulus $\boldsymbol{E}^{\prime}[\mathrm{kPa}]$ & $223.1 \pm 10.4$ & $391.7 \pm 38.4$ \\
\hline tensile loss modulus $\boldsymbol{E}^{n}[\mathrm{kPa}]$ & $8.19 \pm 0.27$ & $38.71 \pm 19.3$ \\
\hline instantaneous elastic modulus $\boldsymbol{E}[\mathrm{kPa}]$ & $223.3 \pm 10.4$ & $394.0 \pm 37.1$ \\
\hline
\end{tabular}

\section{Dynamic stiffening during 3T3-L1 fibroblasts culture}

The previous cell mechanical response data suggested that a fraction of the UVexposed material constitutes a dissipative viscous phase responsible for the absence of spreading of our 2s-exposed Loctite layers. Hence, we decided to culture cells in 2s-exposed Loctite where a round-like shape is preserved and then further crosslink this material to look for a possible application in dynamic mechanical stiffness control for mechanotransduction studies. It has been demonstrated that cells are able to respond to changes in elastic or viscoelastic properties of substrates dynamically in cell culture, and a further cross-linking in Loctite non-polymerized material could then trigger cell spreading and activation of mechanotransduction signals. We cultured fibroblast initially for 24 hours to allow cells to adapt completely to 2 s-exposed Loctite and after this period of time we post-exposed cultured fibroblast to $365 \mathrm{~nm}$ UV light again for $1 \mathrm{~min}$ (photoactivation) and left fibroblasts in culture for an additional 48 hour period to guarantee non toxicity effects caused for a low diffusion of a certain component (Figure 4.A). We analyzed cell viability by calcein AM/PI assay in order to visualized the toxic effects of further polymerization and UV light exposure. After the second UV light exposure, no dead cells or a decrease in cell number was observed after 48 hours of post-culture even more there was a significant increase of 1.9 fold (21 vs 41 cells per area) in cell number (Figure 4.B). Nevertheless, after 48 hours of culture, fibroblasts showed a significant increase in spreading after photoactivation of soft Loctite, compared with the non-photoactivated control substrates, where Loctite was only polymerized for $2 \mathrm{~s}$ prior to cell seeding. Quantification of cell area showed a 2-fold increase in posterior cross-linked Loctite, with the same cell areas found in the previous experiments, suggesting that cells are responding to the second dose as if they were seeded on the 40s-exposed substrate (Figure 4.B). Once we demonstrated the possibility to dynamically tune the mechanical characteristics of Loctite and that 3T3 cells responded favorably, we interrogated the fibroblast whether they activated mechanotransduction signals caused by further spreading. We looked for subcellular 
localization of YAP/TAZ proteins and observed an increase in qualitative . 10.1 chleededicle Online localization of YAP/TAZ after photoactivation and it correlated with the increase of cell spreading described above (Figure 4.C). Data then demonstrate that Loctite photosensitive resin could be considered as a dynamic substrate for mechanotransduction studies as the 3T3 fibroblast clearly responded to a second event of polymerization during cell culture without affecting cell viability and promoting spreading and activation of YAP/TAZ transcriptional cofactors.

A
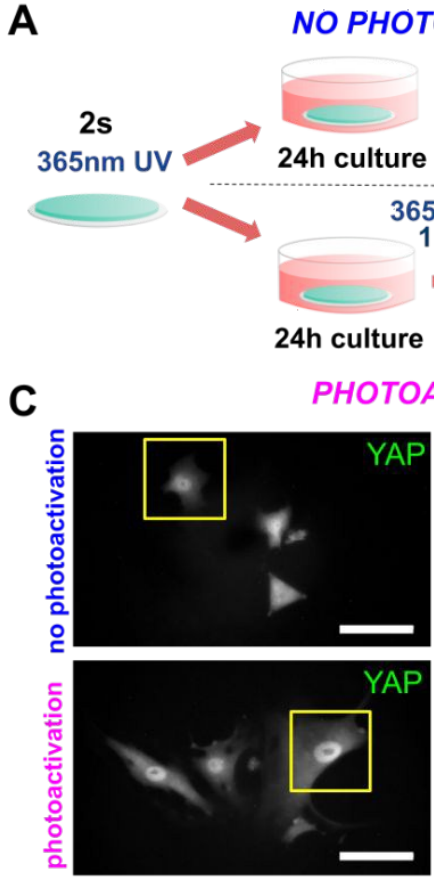

B
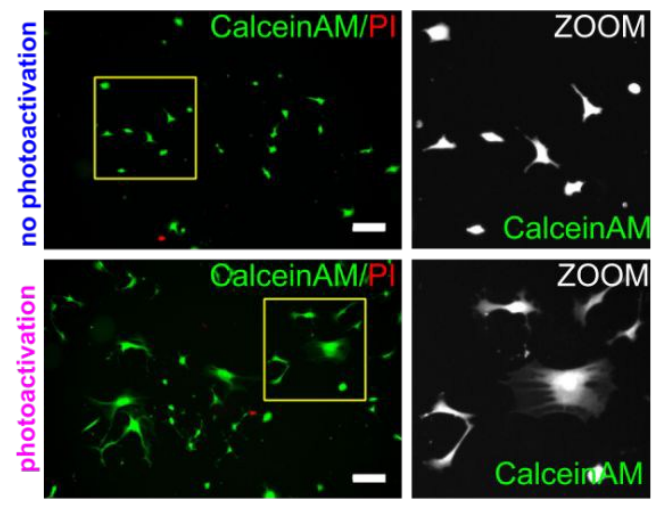

Cell count
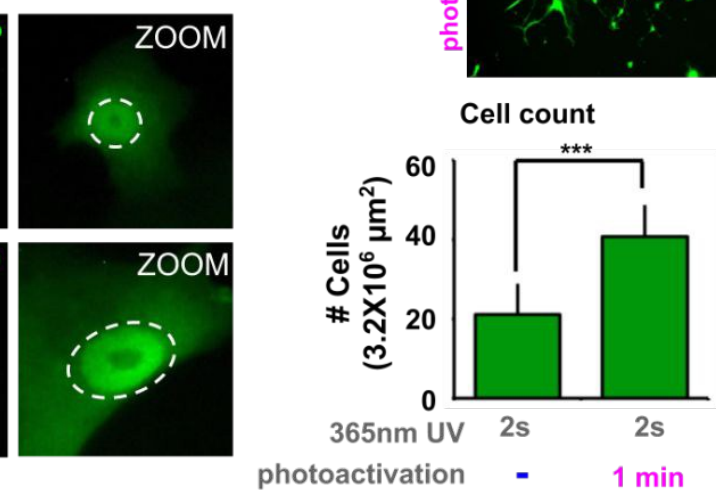

photoactivation - $1 \mathrm{~min}$

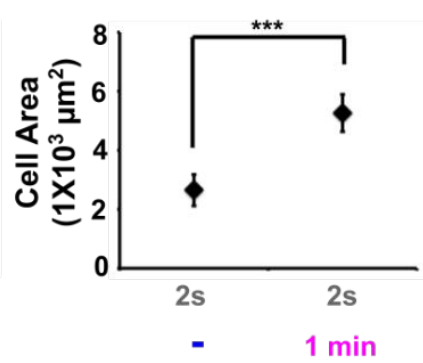

Figure 4. Loctite 3525 as a mechanical tunable photosensitive resin for cell culture platforms. (A) Experimental set up for photoactivation during cell culture. (B) Live/dead assay of 3T3-L1 fibroblasts cultured on 2s UV cross-linked Loctite after a second exposure of UV light. Fibroblasts were cultured for 24 hours then cells were exposed to $365 \mathrm{~nm}$ UV for $1 \mathrm{~min}$ and after that culture was maintained for additional 48 hours. Cell count was presented as a total number of cells in an area of $3.2 \times 10^{6} \mu \mathrm{m}^{2}$. Scale bar $=100 \mu \mathrm{m}$. Data are represented as mean \pm SD of at least 10 different fields from 2 independent experiments. ${ }^{* * *} p<0.0007$ compared with 2 s. Cell spreading analysis was presented as the area detected by calcein AM signal. Data are represented as mean $\pm \mathrm{SD}$ of 10 different fields from 2 independent experiments. ${ }^{* * *} p<0.0001$ compared with $2 \mathrm{~s}$. (C) Nuclear translocation of YAP/TAZ mechanosensitive cofactors in fibroblasts cultured on activated and none activated loctite. YAP/TAZ subcellular localization was detected by immunofluorescence (green); nucleus (dashed circles). Scale bar $=200 \mu \mathrm{m}$.

\section{Microstructuring of Loctite $\mathbf{3 5 2 5}$ for mechanobiology studies}

In mechanobiology, micropillars with specific aspect ratios can be used for Traction Force Microscopy (TFM) ${ }^{30}$. As commented earlier, Loctite 3525 may be readily used to replicate high-resolution micromolds patterns with great fidelity ${ }^{27}$ or can be directly micropatterned using a blu-ray laser ${ }^{28}$ for simple rapid prototyping. We tested the 


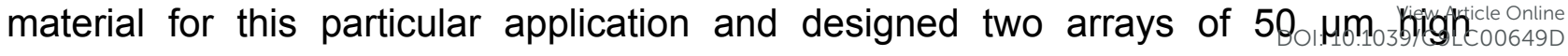
micropillars with a $10 \mu \mathrm{m}$ diameter and a uniform periodicity in both $\mathrm{x}$ and $\mathrm{y}$ directions with different separations of $10 \mu \mathrm{m}$ and $20 \mu \mathrm{m}$, to allow different cell spreading and test whether 3T3-L1 mouse embryonic fibroblasts exerted different forces on micropillars separated by different distances, only as a proof of concept of microstructuring of the glue for TFM applications. It was interesting to use the cured Loctite resin instead of PDMS to construct the micropillars using the soft lithographic replica-molding technique, as PDMS preparation may result in local heterogeneities in mechanical properties 50 . Molds were fabricated in the photosensitive SU8 resin using a conventional photolithography mask and the structures were then successfully replicated in Loctite 3525 crosslinked on top of the silanized mold during $1 \mathrm{~min}$. The replicas obtained from the micromolds showed great fidelity, similar to what is typically obtained with PDMS (Figure 5.A) and the spring constant of these pillars was calculated to be $4.71 \mathrm{nN} / \mu \mathrm{m}$ in average. Micropillars were coated with fibronectin previously to 3T3 fibroblasts seeding on these structures, to measure the range of forces applied on the vertical structures. After 48 hours of culture, some fibroblasts adhered at the top of the micropillars and the samples were finally prepared for scanning electron microscope (SEM) characterization in order to precisely measure the deflection of the pillars.

It is known that cells distribute traction forces along the micropillars on top of which they are sitting. We only considered the adhesions at the top of the micropillars for the analysis of cell traction forces (Figure 5A, white arrow). The average value of exerted force calculated in our proof-of-concept assays (Figure 5.A and 5.B) was of $16 \mathrm{nN}$ for the $10 \mu \mathrm{m}$-separated pillars and $25 \mathrm{nN}$ for the $20 \mu \mathrm{m}$-separated pillars, falling within the range of other reports in the literature for 3 T3 fibroblasts ${ }^{51}$.

We were also interested in detecting focal adhesion to demonstrate that pulling forces exerted by fibroblast could derive directly from them, as it has been shown ${ }^{30}$. The material and structures allowed us to detect vinculin protein and actin filaments in fibroblasts cultured for 48 hours. It was possible to observe punctual marks of vinculin at the very top of the micropillars (Figure 5.C, white arrow). However, we observed also a strong vinculin signal surrounding the flexed micropillars and this accumulated vinculin was colocalizing with an accumulated signal of actin filaments (Figure 5.C, yellow arrow). This colocalization suggests that actomyosin complex was participating in pulling the micropillars in fibroblasts although this requires further testing to conclude. However, these results show that the cost-effective, off-the-shelf Loctite 3525 glue may be employed as an excellent material for mechanobiology studies with Traction Force Microscopy using conventional microfabrication techniques. 
A

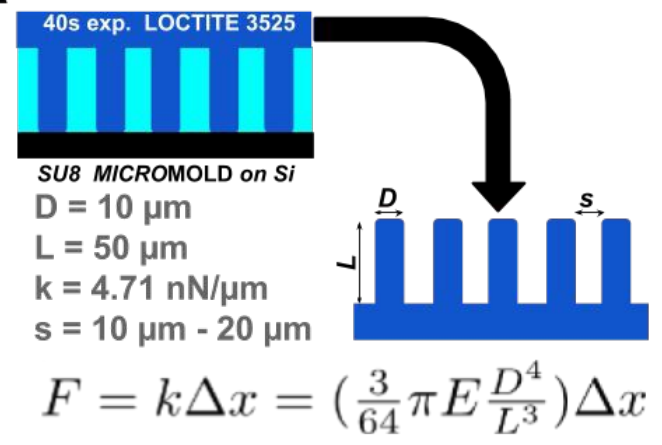

C

Pillars $10 \times 20 \mu \mathrm{m}$
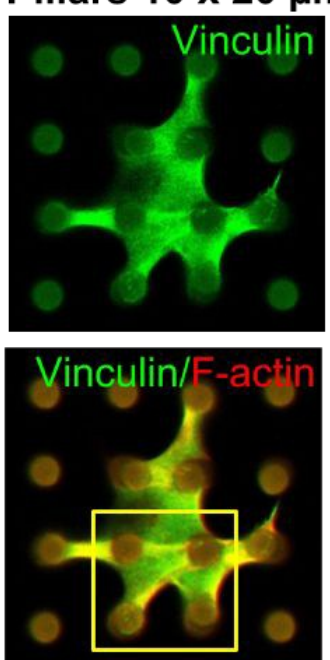
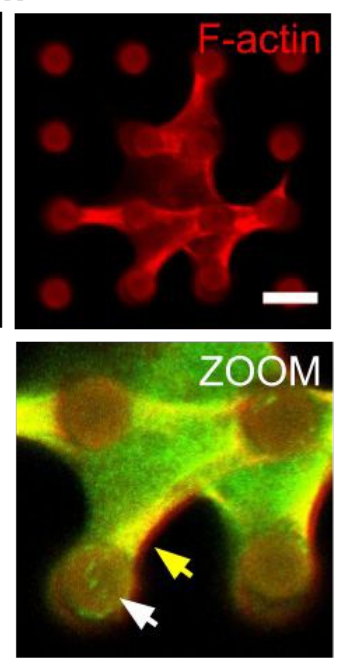

B

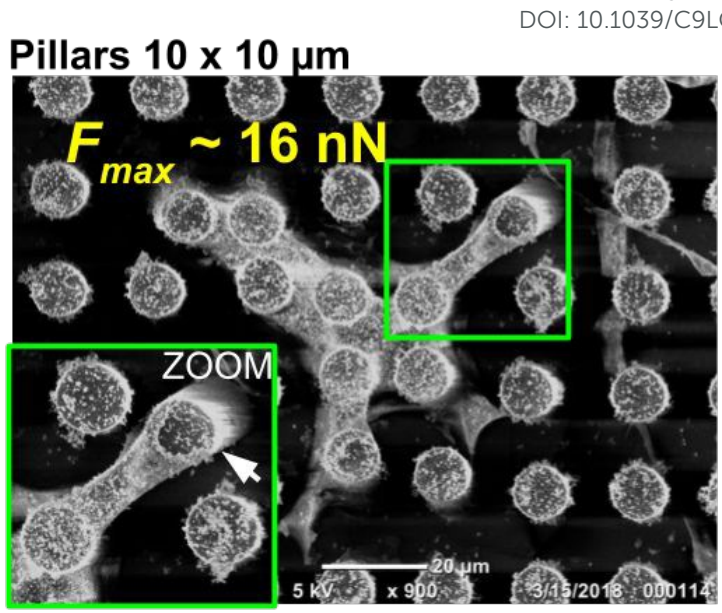

Pillars $10 \times 20 \mu \mathrm{m}$

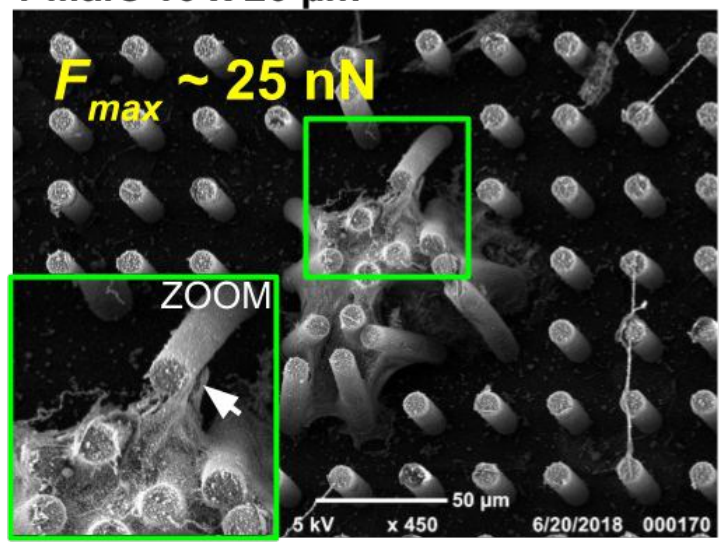

Figure 5. Loctite 3525 as a material for traction force microscopy. (A) Fabrication of UV cured Loctite micropillars. 50 micron high Loctite micropillars with a 10 micron diameter were fabricated with different spacings (10 and 20 microns) using the replica-molding technique to measure the force exerted by 3T3-L1 fibroblasts. Forces were calculated from the deflected micropillars observed in scanning electron microscopy (SEM) micrographs. (B) Deflection of Loctite micropillars. Fibroblasts were cultures on fibronectin-covered micropillars spaced by 10 or $20 \mu \mathrm{m}$. Cells were fixed with paraformaldehyde, contrasted with osmium tetroxide and observed through SEM. White arrows points a deflected micropillar. (C) Focal adhesions in 3T3 fibroblasts cultured on micropillars. Fibroblasts were cultured on fibronectin-covered micropillars spaced by $20 \mu \mathrm{m}$ and FA were detected by immunofluorescence. Vinculin (green); F-actin (red). White arrow points focal adhesions over top of a micropillar and yellow arrow points vinculin recruitment around the micropillar. Scale bar $=20 \mu \mathrm{m}$.

\section{Loctite $\mathbf{3 5 2 5}$ as a adhesion layer for hydrogels}

Interestingly, in this work it was possible to use a couple of droplets of Loctite 3525 as an intermediary adhesion layer for polyacrylamide (PAA) hydrogels on both glass substrates and PDMS slabs without any other particular treatment prior to hydrogel attachment. This made it very attractive to avoid additional surface chemistry steps or even for integration inside chips. PAA hydrogels were polymerized on top of cured Loctite micropatterns using the same recipe as described previously and reported in 
31 and showed an excellent resistance to peel-test (Supporting Information Matseitianticle online Figures S4-S5). Poly (ethylene glycol) diacrylate of PEGDA hydrogels as well PAA hydrogels were also successfully transferred and crosslinked on Loctite using a different method with UV light and Irgacure 2959 as a photoinitiator (see also Supporting Information Material for details). As previously mentioned, polyacrylamidebased hydrogels are commonly used in mechanobiology studies, thanks to the excellent control of stiffness they offer, with a great reproducibility 24,52 . However, most of the gels commonly used in mechanobiology assays do not polymerize nor adhere to glass without previous surface treatment. These treatments usually require toxic or aggressive compounds to attach soft materials and are not ideal. Also, it is difficult to adhere such hydrogels onto plastic substrates and this is limiting the use of soft viscous gels in high throughput assays where large amounts of hydrogels need to be attached to culture plates or in lab-on-chip platforms ${ }^{53,54}$. On the other hand, it is also very impractical to attach hydrogels inside PDMS microfluidic chips as this usually requires a high level of expertise ${ }^{9}$.

In order to validate the use of Loctite 3525 as an adhesion layer for hydrogels on glass or PDMS without previous treatment, we cultured human alveolar adenocarcinoma A549 cell line as well as 3T3-L1 on $500 \mathrm{~Pa}$ and $20 \mathrm{kPa}$ PAA hydrogels fabricated using the protocol previous described in ${ }^{8}$. We used collagen type I to promote cell adhesion on the gels, crosslinked using acrylic acid NHS-ester ${ }^{24}$. We analyzed cell spreading by detecting actin filaments. As we expected, no spreading was observed in cells seeded on 500 Pa PAA hydrogels and A549 cells showed cortical actin instead (Figure $6 . B$, white arrow). It is known that confined epithelial cells present a cortical actin cytoskeleton ${ }^{55,56}$. On the other hand, A549 cells cultured on $20 \mathrm{kPa}$ PAA did spread, showing some stress fibers (Figure 6.B, yellow arrow). In 3T3-L1, we detected F-actin and localization of YAP/TAZ proteins as mechanotransducers. We observed that fibroblast showed an increase in spreading in stiff $(20 \mathrm{kPa})$ than soft $(1 \mathrm{kPa})$ substrates and, according with this effect, YAP/TAZ proteins translocated to cytoplasm in $1 \mathrm{kPa}$ PAA hydrogels (Supporting Material Figure 7). So, the use of Loctite photosensitive resin is presented as an alternative method to polymerized PAA and PEGDA hydrogels (data not shown) on glass and PDMS substrates by using intermediate cured Loctite layers. 


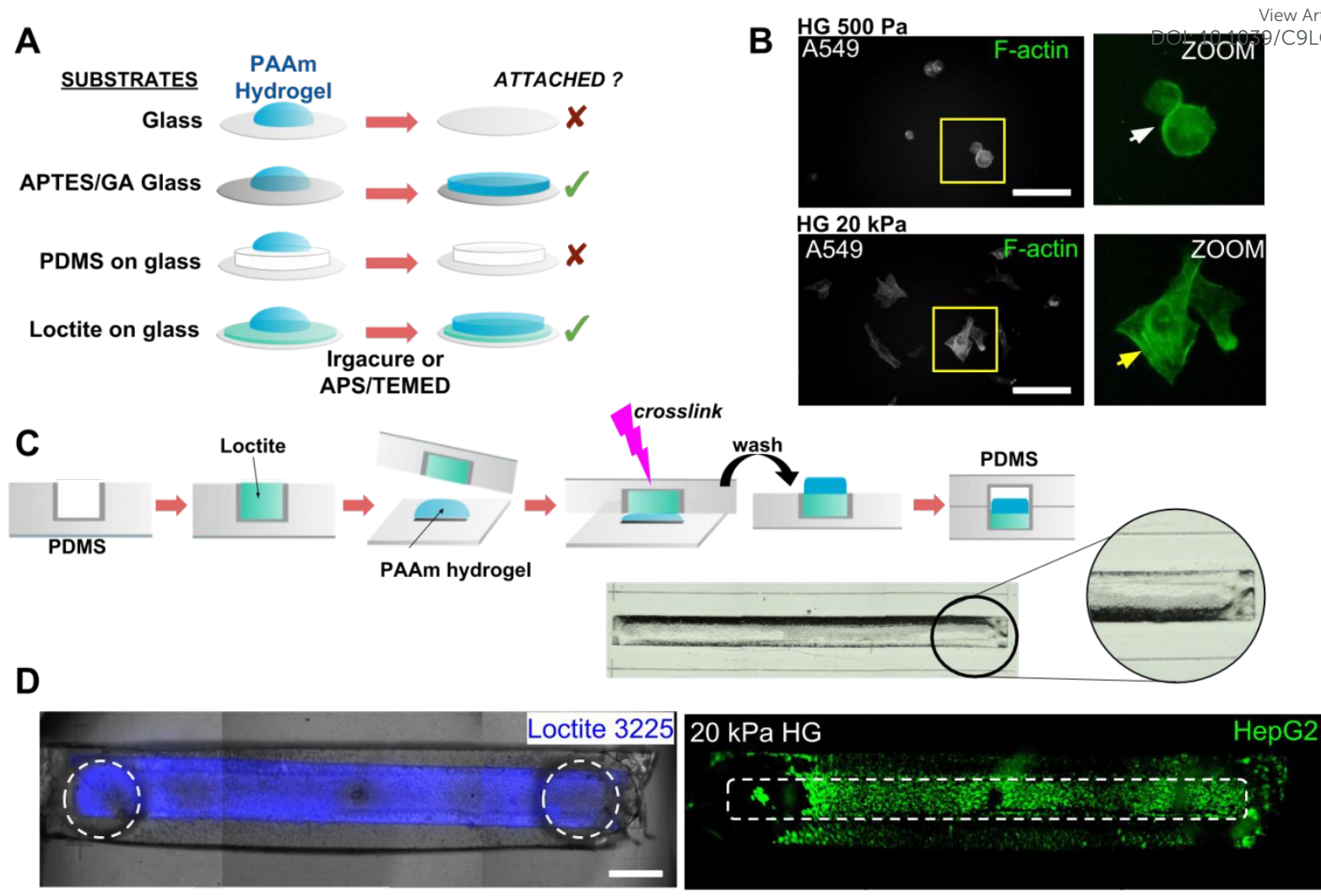

Figure 6. Loctite 3525 photosensitive resin is a suitable material for cellular scaffolds fabrication. (A) Representative adhesion properties of different treated glass surfaces to polyacrylamide (PAA) hydrogels. Coverslips were covered with APTES/glutaraldehyde, polydimethylsiloxane (PDMS) and 40s UV exposed Loctite 3525. (B) Culture of human lung epithelial cell line A549 on PAA hydrogels (HG) with different stiffness. $500 \mathrm{~Pa}$ and $20 \mathrm{kPa}$ PAA HG were polymerized on Loctited-covered coverslip. Rat tail collagen type I was cross-linked to PAA hydrogels as an adhesion promoter. F-actin was detected by Alexa Fluor 488 phalloidin (green); white arrow points cortical actin and yellow arrow points stress actin fibers. Scale bar $=200 \mu \mathrm{m}$. (C) Fabrication set up of one-channel chip covered on the surface with PAA HG. (D) Culture of human liver hepatocellular carcinoma cell line HepG2 in one-channel biomimetic chip. PAA HG was only polymerized over the Loctite-covered surface inside the channel (blue). Collagen type I was cross-linked to acrylamide for promoting cell adhesion and live HepG2 cells were detected by using calcein AM (green) after 24 hours of culture. Scale bar is $1000 \mu \mathrm{m}$.

We then studied the possible use of Loctite 3525 in the fabrication of cell scaffolds integrating hydrogels inside PDMS microfluidic channel. Similarly to what was presented previously, Loctite was used as a substrate to allow PAA polymerization and attachment while PDMS oxygen inhibition layer inhibited gels crosslinking outside Loctite, thus patterning hydrogels selectively only on top of Loctite inside a PDMS chip (Figure 6). A microfluidic chip was designed for cell culture, with a central channel covered with $20 \mathrm{kPa}$ PAA hydrogel (several orders of magnitude softer than PDMS) using a simple procedure described in Figure 6 (photograph details are shown in Supporting Information Material Figure S4). To prove that the gel was functional for integration inside a microfluidic chip, a human liver carcinoma cell line, HepG2, was seeded and cultured inside the channel for 24 hours. It is known to be a delicate cell 
line requiring the presence of a proper protein (collagen type I) to attachol. We calcein AM to visualise live cells in the channel and we observed a strong signal in HepG2 cells (Figure 5.D). We have also tested this procedure for different gels and it was possible to cross-link hydrogels using UV and Irgacure 2959 as a photoinitiator, proving the versatility of Loctite to integrate hydrogels inside PDMS. Finally, a more complex PDMS-based microfluidic chip using selective hydrogel attachment between two microfluidic chamber has also been successfully fabricated (see Supporting Information Material Figure S6) but its application as diffusion microbarrier for coculture will be reported elsewhere. All this allowed us to demonstrate that the Loctite 3525 photosensitive resin is a very useful material for PDMS chips fabrication with stiffness control using integrated hydrogels. It is also compatible with simple softlithography techniques, compared with other reported methods and although some precautions need to be respected (see Supporting Information Material Figure S7), it enables the simple integration of gels inside PDMS for mechanobiology assays.

\section{Conclusions}

In this work, it was shown that the commercial Loctite 3525 photosensitive glue is an excellent material for mechanobiology assays. After confirming that this methacrylatebased resin that uses TPO photoinitiator was biocompatible, we demonstrated that a stiffness tuning is possible by controlling the crosslinking UV dose and exposure time. We observed that 3T3-L1 mouse embryonic fibroblasts seeded on cured Loctite 3525 layers responded to different exposure times, evidenced by different spreading areas, and that they even remained round-shaped at the shortest exposure time. This behavior was unexpected for fibroblasts on a material with an elastic modulus in the $\mathrm{MPa}$ range ${ }^{22}$. We thus characterized further the material and exhibited viscous dissipation properties by measuring relaxation modulus, a phenomenon that may cause the absence of spreading by modifying the immediate apparent substrate stiffness sensed by cells. This has been suggested very recently, also in 3T3 fibroblasts, but with softer hydrogels, with a shear storage elastic modulus G' of $5 \mathrm{kPa}$ and variable G" moduli between 0 and $500 \mathrm{~Pa}{ }^{24}$. After observing that cured Loctite 3525 presented different viscous dissipation behaviors depending on the UV exposure time, we used a generalized Maxwell model including elastic spring and viscous dashpot in parallel with equilibrium, long-range elastic spring to describe the crosslinked material at different exposure times and we calculated all relevant mechanical properties in order to compare relaxation timescales of the viscous part of the material in order to understand what apparent stiffness was perceived by 3T3 fibroblasts and determined whether it would spread or not, following the model presented recently in 25 .

Understanding this phenomenon better, we used less-crosslinked Loctite 3525 samples and modified the material stiffness after fibroblasts were already cultured on it. As expected, fibroblasts responded to the change in mechanical properties by spreading after the stimulation, confirming that the material can be used for dynamic 
mechanical changes with a simple control of light exposure. This is veryoimporitalanticle Online when dynamic mechanotransduction processes inside the cell are studied while stiffness is increased ${ }^{25,29}$.

Loctite 3525 has also been used in this work to confirm its potential as a 3D microstructured material applicable to studies of the impact of topology in cell culture. In particular, we tested micropillars fabrication for studies in traction force microscopy to measure cell force using such patterns ${ }^{30}$. The photocurable resin resulted excellent for the straightforward replication of previously designed SU8 micromolds with pillars of controlled aspect ratios; the forces measured here, in 3T3-L1 fibroblasts, were comparable with the literature, proving the material is also a very good candidate for low-cost rapid prototyping of such structures.

Finally, another interesting field of application of Loctite 3525 is as a very strong adhesion layer for hydrogels, stable and useful for many cell types, without the need for any additional surface treatment. We found that the material may be cured on plastics, PDMS and glass substrates onto which it adhered very well and then hosted hydrogels without further pre-treatment. This is particularly important when mechanobiology assays are required in PDMS microfluidic chips where low Young's moduli are required ${ }^{9}$. With a peel-test performed with a custom-made traction force measurement system, we show that adhesion of polyacrylamide layers attached to Loctite is much more resistant than on other substrates treated with common techniques to fix the gels. The Loctite-hydrogel constructs also resisted immersion in aqueous media for prolonged periods of time with no affectation of transparency or surface roughness, and without detachment.

As all these experiments are straightforward to perform, with a ready-to-use commercial material, Loctite 3525 is an excellent material to consider to provide a lot of interesting information in mechanobiology, without the need to involve complex processes and with a greater guarantee of reproducibility as no complex fabrication processes are involved at any time.

\section{Authors contributions}

G. V.-V., and M. H. designed all the experiments. G. V.-V., and B. D.-B. designed, performed and analyzed all biological experiments; A. J.-E., performed and analyzed biological experiments in polyacrylamide hydrogels; C. P.-G., M. C.-J., and M. H. designed and analyzed all the experiments of fabrication, materials characterization and microstructuring; R. C., and L. M. fabricated the SU8 molds and used to replicate micropillars in Loctite; D. Z.-H., and D. P.-C. performed mechanical characterizations; D. P.-C., and M. H. analyzed mechanical characterization results; S. E.-M., and R. L.M., prepared the samples for SEM characterization. G. V.-V., and M. H. wrote the manuscript.

\section{Acknowledgements}

The authors would like to thank Dr. Ma. Pilar-Carreón and Dr. Violeta Álvarez-Márquez from Instituto de Ciencias Nucleares, UNAM for their help in UV-Vis and FTIR 
measurements. We would also like to thank LaNSBioDyT National Laboratory vaingadicle Online CONACyT-ANR ECOS project for mobility \#276897) and DGAPA-PAPIIT projects \#IT102017 and \#IA206818 for funding. This work was also supported by the French RENATECH network and was partly supported as part of the MuliFAB project funded by FEDER European Regional Funds and Région Occitanie (France) (grant number 16007407/MP0011594)

\section{References}

1 J. Eyckmans, T. Boudou, X. Yu and C. S. Chen, Developmental Cell, 2011, 21, 35-47.

2 K. A. Jansen, D. M. Donato, H. E. Balcioglu, T. Schmidt, E. H. J. Danen and G. H. Koenderink, Biochim. Biophys. Acta, 2015, 1853, 3043-3052.

3 W. J. Polacheck and C. S. Chen, Nat. Methods, 2016, 13, 415-423.

4 L. Li, J. Eyckmans and C. S. Chen, Nat. Mater., 2017, 16, 1164-1168.

5 M. Darnell and D. J. Mooney, Nature Materials, 2017, 16, 1178-1185.

6 J.-W. Shin and D. J. Mooney, Cell Stem Cell, 2016, 18, 16-19.

7 M. Ahearne, Interface Focus, 2014, 4, 20130038.

8 A. J. Engler, S. Sen, H. L. Sweeney and D. E. Discher, Cell, 2006, 126, 677689.

9 N. Annabi, Š. Selimović, J. P. Acevedo Cox, J. Ribas, M. Afshar Bakooshli, D. Heintze, A. S. Weiss, D. Cropek and A. Khademhosseini, Lab Chip, 2013, 13, 3569-3577.

10 H. Wolfenson, B. Yang and M. P. Sheetz, Annu. Rev. Physiol., 2019, 81, 585605.

11 A. Elosegui-Artola, X. Trepat and P. Roca-Cusachs, Trends Cell Biol., 2018, 28, 356-367.

12 A. Elosegui-Artola, R. Oria, Y. Chen, A. Kosmalska, C. Pérez-González, N. Castro, C. Zhu, X. Trepat and P. Roca-Cusachs, Nat. Cell Biol., 2016, 18, 540548.

13 R. Oria, T. Wiegand, J. Escribano, A. Elosegui-Artola, J. J. Uriarte, C. MorenoPulido, I. Platzman, P. Delcanale, L. Albertazzi, D. Navajas, X. Trepat, J. M. García-Aznar, E. A. Cavalcanti-Adam and P. Roca-Cusachs, Nature, 2017, 552, 219-224.

14 J. Swift and D. E. Discher, J. Cell Sci., 2014, 127, 3005-3015.

15 S. E. Szczesny and R. L. Mauck, J. Biomech. Eng., , DOI:10.1115/1.4035350.

16 T. P. Driscoll, B. D. Cosgrove, S.-J. Heo, Z. E. Shurden and R. L. Mauck, Biophys. J., 2015, 108, 2783-2793.

17 E. N. Olson and A. Nordheim, Nat. Rev. Mol. Cell Biol., 2010, 11, 353-365.

18 S. Dupont, L. Morsut, M. Aragona, E. Enzo, S. Giulitti, M. Cordenonsi, F. Zanconato, J. Le Digabel, M. Forcato, S. Bicciato, N. Elvassore and S. Piccolo, Nature, 2011, 474, 179-183.

19 M. Aragona, T. Panciera, A. Manfrin, S. Giulitti, F. Michielin, N. Elvassore, S. Dupont and S. Piccolo, Cell, 2013, 154, 1047-1059.

20 A. Elosegui-Artola, I. Andreu, A. E. M. Beedle, A. Lezamiz, M. Uroz, A. J. Kosmalska, R. Oria, J. Z. Kechagia, P. Rico-Lastres, A.-L. Le Roux, C. M. Shanahan, X. Trepat, D. Navajas, S. Garcia-Manyes and P. Roca-Cusachs, Cell, 2017, 171, 1397-1410.e14.

21 J. H.-C. Wang, B. P. Thampatty, J.-S. Lin and H.-J. Im, Gene, 2007, 391, 1-15. 
22 J. Solon, I. Levental, K. Sengupta, P. C. Georges and P. A. Janmey, Biophyłş J., 2007, 93, 4453-4461.

23 N. Strohmeyer, M. Bharadwaj, M. Costell, R. Fässler and D. J. Müller, Nat. Mater., 2017, 17, 103.

24 E. E. Charrier, K. Pogoda, R. G. Wells and P. A. Janmey, Nat. Commun., 2018, 9, 449.

25 Z. Gong, S. E. Szczesny, S. R. Caliari, E. E. Charrier, O. Chaudhuri, X. Cao, Y. Lin, R. L. Mauck, P. A. Janmey, J. A. Burdick and V. B. Shenoy, Proc. Natl. Acad. Sci. U. S. A., 2018, 115, E2686-E2695.

26 M. Bennett, M. Cantini, J. Reboud, J. M. Cooper, P. Roca-Cusachs and M. Salmeron-Sanchez, Proc. Natl. Acad. Sci. U. S. A., 2018, 115, 1192-1197.

27 R. Delille, M. G. Urdaneta, S. J. Moseley and E. Smela, Journal of Microelectromechanical Systems, 2006, 15, 1108-1120.

28 A. Cruz-Ramírez, R. Sánchez-Olvera, D. Zamarrón-Hernández, M. Hautefeuille, L. Cabriales, E. Jiménez-Díaz, B. Díaz-Bello, J. López-Aparicio, D. PérezCalixto, M. Cano-Jorge and G. Vázquez-Victorio, Micromachines (Basel), , DOI:10.3390/mi9040187.

29 S. R. Caliari, M. Perepelyuk, B. D. Cosgrove, S. J. Tsai, G. Y. Lee, R. L. Mauck, R. G. Wells and J. A. Burdick, Sci. Rep., 2016, 6, 21387.

30 J. L. Tan, J. Tien, D. M. Pirone, D. S. Gray, K. Bhadriraju and C. S. Chen, Proc. Natl. Acad. Sci. U. S. A., 2003, 100, 1484-1489.

31 J. R. Tse and A. J. Engler, Curr. Protoc. Cell Biol., 2010, Chapter 10, Unit 10.16.

32 B. T. Hong, K. S. Shin and D. S. Kim, Journal of Applied Polymer Science, 2005, 98, 1180-1185.

33 N. Guz, M. Dokukin, V. Kalaparthi and I. Sokolov, Biophys. J., 2014, 107, 564575.

34 Y. Ding, G.-K. Xu and G.-F. Wang, Sci. Rep., 2017, 7, 45575.

35 E. M. Chandler, C. M. Berglund, J. S. Lee, W. J. Polacheck, J. P. Gleghorn, B. J. Kirby and C. Fischbach, Biotechnol. Bioeng., 2011, 108, 1683-1692.

36 N. Shoham, R. Gottlieb, O. Sharabani-Yosef, U. Zaretsky, D. Benayahu and A. Gefen, Am. J. Physiol. Cell Physiol., 2012, 302, C429-41.

37 D. Mazia, G. Schatten and W. Sale, J. Cell Biol., 1975, 66, 198-200.

38 L. Escutia-Guadarrama, G. Vázquez-Victorio, D. Martínez-Pastor, B. NietoRivera, M. Sosa-Garrocho, M. Macías-Silva and M. Hautefeuille, Journal of Tissue Engineering, 2017, 8, 204173141774150.

39 S.-Y. Tee, J. Fu, C. S. Chen and P. A. Janmey, Biophys. J., 2011, 100, L25-7.

40 S. S. Desai, J. C. Tung, V. X. Zhou, J. P. Grenert, Y. Malato, M. Rezvani, R. Español-Suñer, H. Willenbring, V. M. Weaver and T. T. Chang, Hepatology, 2016, 64, 261-275.

41 R. J. Albertini, Regul. Toxicol. Pharmacol., 2017, 84, 77-93.

42 J. L. Balestrini, S. Chaudhry, V. Sarrazy, A. Koehler and B. Hinz, Integr. Biol. , 2012, 4, 410-421.

43 J. D. Mih, A. Marinkovic, F. Liu, A. S. Sharif and D. J. Tschumperlin, J. Cell Sci., 2012, 125, 5974-5983.

44 P. P. Provenzano and P. J. Keely, J. Cell Sci., 2011, 124, 1195-1205.

45 B. Stutchbury, P. Atherton, R. Tsang, D.-Y. Wang and C. Ballestrem, J. Cell Sci., 2017, 130, 1612-1624.

46 S. Dupont, Experimental Cell Research, 2016, 343, 42-53.

47 S. E. Ross, Science, 2000, 289, 950-953. 
48 C. Christodoulides, C. Lagathu, J. K. Sethi and A. Vidal-Puig, Trends ind view Article Online Endocrinology \& Metabolism, 2009, 20, 16-24.

49 L. Cacopardo, N. Guazzelli, R. Nossa, G. Mattei and A. Ahluwalia, J. Mech. Behav. Biomed. Mater., 2019, 89, 162-167.

50 A. J. S. Ribeiro, A. K. Denisin, R. E. Wilson and B. L. Pruitt, Methods, 2016, 94, 51-64.

51 H. Jeon, E. Kim and C. P. Grigoropoulos, Biomedical Microdevices, 2011, 13, 107-115.

52 C. E. Kandow, P. C. Georges, P. A. Janmey and K. A. Beningo, Methods Cell Biol., 2007, 83, 29-46.

53 E. J. Semler, P. A. Lancin, A. Dasgupta and P. V. Moghe, Biotechnol. Bioeng., 2005, 89, 296-307.

54 J. D. Mih, A. S. Sharif, F. Liu, A. Marinkovic, M. M. Symer and D. J. Tschumperlin, PLoS One, 2011, 6, e19929.

55 C. C. DuFort, M. J. Paszek and V. M. Weaver, Nat. Rev. Mol. Cell Biol., 2011, 12, 308-319.

56 M. Bosch-Fortea and F. Martín-Belmonte, Curr. Opin. Cell Biol., 2018, 50, 4249. 